\title{
Application of photoconductivity decay and photocurrent generation methods for determination of minority carrier lifetime in silicon
}

\author{
S N SINGH*, R GANDOTRA, P K SINGH and B C CHAKRAVARTY \\ Division of Electronic Materials, National Physical Laboratory, New Delhi 110 012, India
}

\begin{abstract}
Minority carrier lifeline, $\tau$, is one of the most important parameters which has a decisive effect on the performance of silicon devices based on excess carriers. The value of $\tau$ is greatly affected by the presence of impurities and defects in silicon and its value provides a fair indication of quality of the material. Photoconductivity decay (PCD) and photocurrent generation (PCG) methods are simple and low cost methods of measurement of minority carrier lifetime in silicon wafers. However, their application requires care. The PCD method can give quite misleading results in case of polycrystalline wafers if there exists potential barriers at the grain boundaries which may affect majority carrier mobility significantly. PCG needs creation of an induced $p^{+}-p-n^{+}$structure of substantially good quality that should not degrade with time. For PCG method the $\tau$ measurement under vacuum conditions provides correct and consistent results.
\end{abstract}

Keywords.

\section{Introduction}

Silicon is one of the most pure materials produced by mankind. Still a significantly large concentration of impurities may always be present in it. Single crystal silicon $(c-\mathrm{Si})$ is the backbone of the electronic industry. The electronic properties of $c$-Si are very sensitive to the presence of impurities. In most cases $c$-Si requires to have a limited concentration of desired or dopant impurities which control the resistivity. Besides this there may be a significant concentration of undesired impurities and defects that give rise to recombination centres which control the minority carrier lifetime $(\tau)$ and have decisive influence on the performance of the silicon devices based on excess carriers.

It is well known that the silicon single crystals prepared by floatzone (FZ) method have much less concentration of many undesired impurities particularly oxygen and carbon than the $\mathrm{Cz}$ grown single crystal silicon. This results in much higher value of $\tau$ in the FZ-Si wafers than in the $\mathrm{Cz}-\mathrm{Si}$ wafers. In most cases it is desirable to have the knowledge of the minority carrier lifetime in the material. Photoconductivity decay has been the most widely used method to measure lifetime in silicon wafers. In the following we shall discuss the essential features of the PCD method and also the photocurrent generation (PCG) method.

The minority carrier lifetime provides information on how fast or slow the recombination of the minority carriers takes place in that semiconductor material. In silicon its value depends on the concentration of the recombination

*Author for correspondence (snsingh@ mail.nplindia.ernet.in) centres, $N_{\mathrm{R}}$, their capture cross-section, $\sigma_{\mathrm{c}}$, for minority carriers and the average thermal velocity $\left\langle v_{\mathrm{th}}\right\rangle$ of the carriers.

Mathematically, it is given by

$$
\tau=\frac{1}{N_{\mathrm{R}} \sigma_{\mathrm{c}}<v_{\mathrm{th}}>} .
$$

Since impurities present in the semiconductor lead to creation of recombination centres directly or by causing defects in the semiconductor, the value of $\tau$ is intimately related with the concentration of the impurities. Alternately the value of $\tau$ provides a direct assessment of the quality of the material for various device applications. Figure 1 shows the dependence of $\tau$ on dopant impurity concentration in $c$-Si grown by different techniques (Ciszek). It shows that $\tau$ decreases drastically as the impurity concentration increases beyond $10^{17} \mathrm{~cm}^{-3}$.

\section{Photoconductivity decay method}

In practice, PCD method is applied in two ways. In first case, it requires forming of two ohmic contacts on the specimen separated by a few mm wide rectangular polished silicon region through which a constant current is passed. The photoconductivity, $\Delta \sigma$, is represented by a voltage change, $\Delta V$, in the ohmic voltage drop across the specimen. In the second case, the PCD method does not require ohmic contacts but generally uses microwave power reflection based detection of the change in photoconductivity with time (Citarella et al 2002). It is an attractive nondestructive method of $\tau$ measurement but requires a costly set up. We shall discuss the former in detail. Henceforth, PCD would mean the PCD with ohmic con- 
tacts unless specified otherwise. In PCD method, two ohmic contacts separated by a distance, $d$, are made on the same side of the wafer as shown in figure 2 .

The exposed silicon surface between the contacts is polished and is passivated to minimize the surface recombination. A constant current $(I)$ is passed through the two contacts and the voltage drop, $V_{0}$, between them is measured which is given by

$$
V_{0}=\frac{I d}{A \sigma_{0}},
$$

where $\sigma_{0}$ is the dark conductivity of the specimen and $A$ the area of cross-section through which the current $(I)$ passes.

The wafer is illuminated with a suitable wavelength, which gets absorbed in the silicon wafers and enhances the wafer conductivity to $\sigma$. When the illumination is terminated abruptly the decay of the voltage, $\Delta V$, between the contacts is measured as a function of time which is given by

$$
\Delta V=\frac{I d}{A \sigma_{0}} \frac{\Delta \sigma}{\sigma},
$$

where

$$
\Delta \sigma=\sigma-\sigma_{0} .
$$

For small intensities of light pulse, $\sigma_{0}$ being sufficiently high, $\Delta \sigma \ll \sigma_{0}$ which represents the case of a low level condition for $c$-Si and thus (2) modifies as

$$
\Delta V=\frac{I d}{A \sigma_{\mathrm{g}}} \frac{\Delta \sigma}{\sigma_{0}}
$$

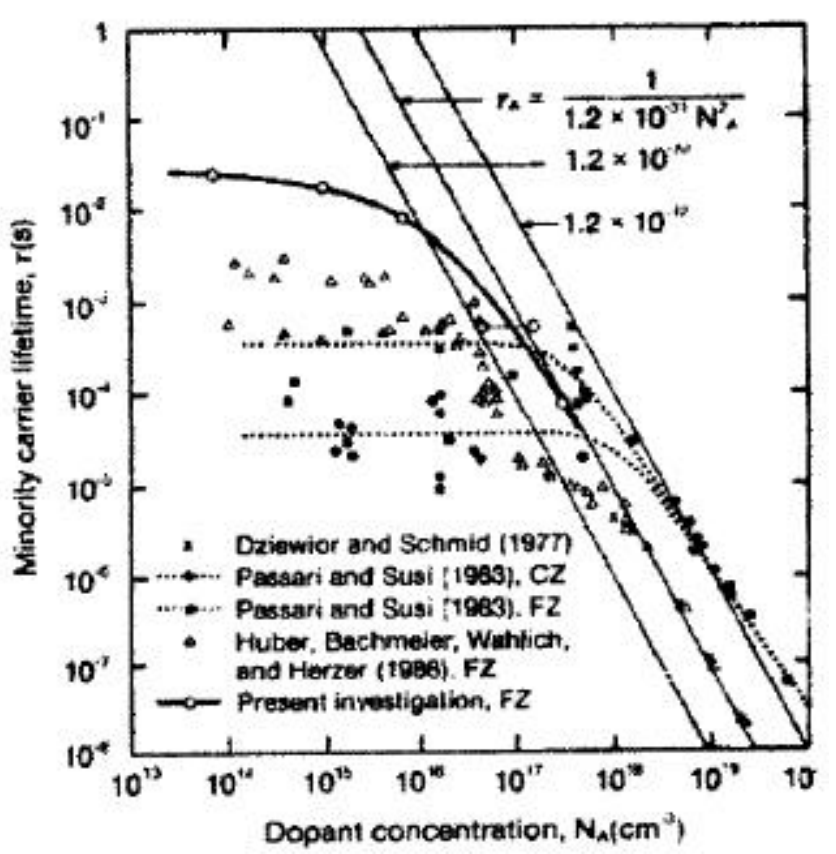

Figure 1. Dependence of minority carrier lifetime on dopant concentration in single crystal silicon (after Ciszek).
Under this condition, the change, $\Delta V$, in voltage is governed by the decay of $\Delta \sigma$. Therefore, the decay of $\Delta V$ with time is portrayed essentially by the decay of the photoconductivity, $\Delta \sigma$.

\subsection{Photoconductivity of n-type polycrystalline silicon under low level conditions}

We will discuss the case of $n$-type polycrystalline silicon although the analysis can be applied equally well to $p$ type polycrystalline silicon also. In case of polycrystalline silicon there exists potential barriers, $V_{\mathrm{Bo}}$, across grain boundaries which are formed due to trapping of majority carriers (electrons) at the grain boundaries. As a result the electron mobility is given by

$$
\mu_{\text {no }}=\mu_{\mathrm{g}}\left\{1+\frac{\mu_{\mathrm{g}}}{\mu_{0}} \exp \left(\frac{q V_{\mathrm{Bo}}}{k T}\right)\right\}^{-1},
$$

where $q, k$ and $T$ have their usual meanings. $\mu_{\mathrm{g}}$ is the majority carrier mobility in the grains and $\mu_{0}$ is defined as

$$
\mu_{0}=\frac{q d_{\mathrm{g}}}{\sqrt{2 \pi m_{\mathrm{e}}^{*} k T}},
$$

where $d_{\mathrm{g}}$ is the grain diameter and $m_{\mathrm{e}}^{*}$ the effective electron mass. Potential barrier, $V_{\mathrm{B}}$, decreases under illumination and with increase in temperature as shown in figures 3 and 4.

Under illumination the barrier height is reduced to $V_{\mathrm{B}}$ and then the mobility, $\mu_{\mathrm{n}}$, is given by

$$
\mu_{\mathrm{n}}=\frac{\mu_{\mathrm{g}}}{1+\frac{\mu_{\mathrm{g}}}{\mu_{0}} \exp \frac{q V_{\mathrm{B}}}{k T}} .
$$

In terms of carrier density and mobility, $\sigma_{0}$ and $\sigma$ can be expressed as

$$
\begin{aligned}
& \sigma_{0}=q\left(n_{0} \mu n_{0}+p_{0} \mu p_{0}\right), \\
& \sigma=q\left(n \mu_{\mathrm{n}}+p \mu_{\mathrm{n}}\right),
\end{aligned}
$$

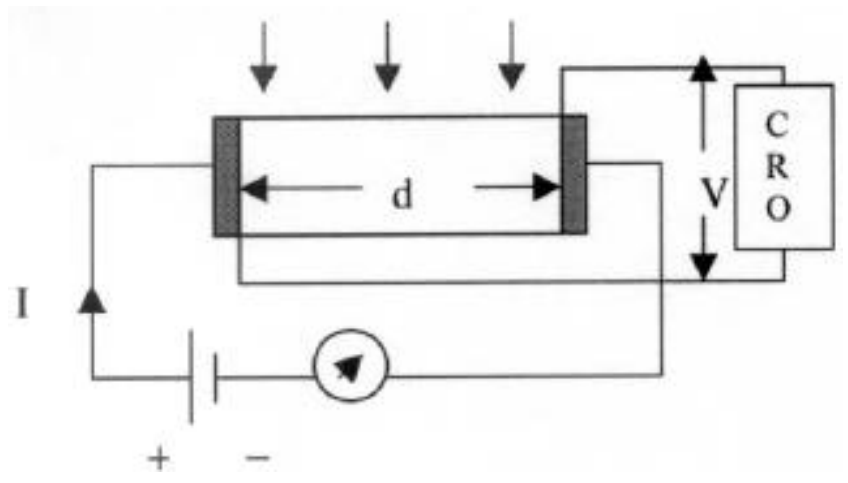

Figure 2. Schematic diagram of PCD set up for $\tau$ measurement. 
where $n_{0}$ and $p_{0}$ are background electron and hole density, $\mu_{\mathrm{n} 0}$ and $\mu_{\mathrm{p} 0}$ are background electron and hole mobility. $n$ and $p$ are modified electron and hole density due to photoconductivity. $\mu_{\mathrm{n}}$ and $\mu_{\mathrm{p}}$ are modified electron and hole mobility due to photoconductivity.

We define

$$
\begin{aligned}
& n=n_{0}+\Delta n, p=p_{0}+\Delta p, \\
& \mu_{\mathrm{n}}=\mu_{\mathrm{n} 0}+\Delta \mu n, \mu_{\mathrm{p}}=\mu_{\mathrm{p} 0}+\Delta \mu_{\mathrm{p}},
\end{aligned}
$$

where $\Delta n$ and $\Delta p$ represent the changes due to photoconductivity in electron and hole density and $\Delta \mu_{\mathrm{n}}$ and $\Delta \mu_{\mathrm{p}}$ represent changes in electron and hole mobility.

This gives $\Delta \sigma$ as

$$
\Delta \sigma=q\left(n_{0} \Delta \mu_{\mathrm{n}}+p_{0} \Delta \mu_{\mathrm{p}}\right)+q \Delta p\left(\mu_{\mathrm{n}}+\mu_{\mathrm{p}}\right) .
$$

In the case of large grain $n$-type polycrystalline silicon (multicrystalline), generally, $n_{0} \gg p_{0}$ and $n_{0} \gg \Delta p$. Under these conditions, (10) reduces to

$$
\Delta \sigma=q n_{0} \Delta \mu_{\mathrm{n}}+q \Delta p\left(\mu_{\mathrm{n}}+\mu_{\mathrm{p}}\right) .
$$

In (11) the first term on right hand side (RHS) represents the photoconductivity $\left(\Delta \sigma_{\mathrm{mc}}\right)$ due to the change in majority carrier mobility, whereas, the second term represents photoconductivity $\left(\Delta \sigma_{\mathrm{exc}}\right)$ due to the excess carriers. Mathematically, we can represent (11) as

$$
\Delta \sigma=\Delta \sigma_{\mathrm{mc}}+\Delta \sigma_{\mathrm{exc}} .
$$

Essentially $\Delta \sigma_{\mathrm{mc}}$ is an effect of excess carrier recombination at grain boundaries under illumination which leads to the decrease in the potential barrier, $V_{\mathrm{B}}$ and thereby increases the majority carrier mobility.

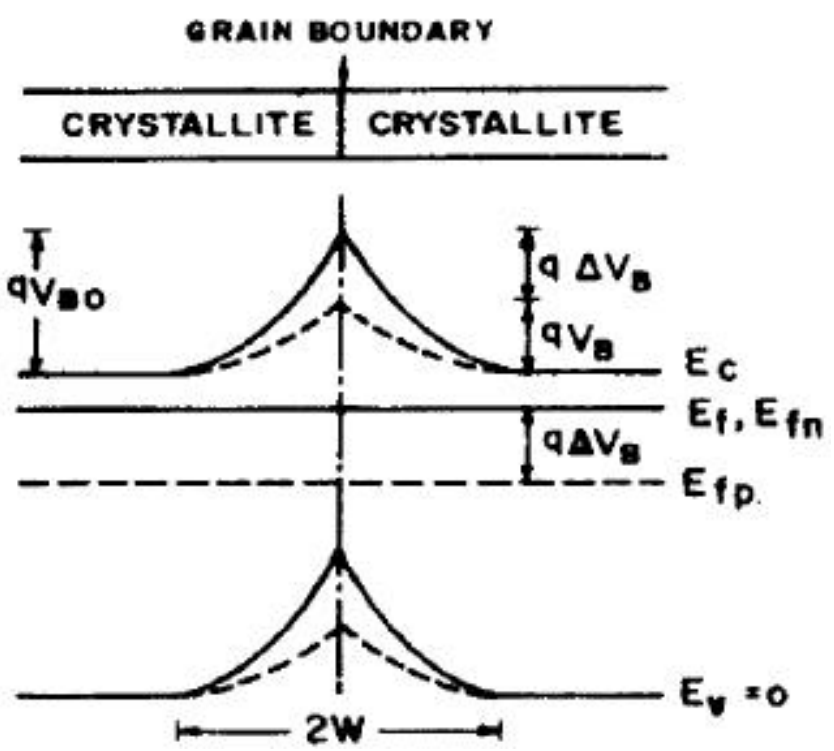

Figure 3. Effect of illumination on the grain boundary potential barrier in $n$-type polycrystalline silicon.
The relative dominance of $\Delta \sigma_{\mathrm{mc}}$ and $\Delta \sigma_{\mathrm{exc}}$ as a function of carrier generation rate is depicted in figure 5 . It can be seen that for smaller generation rates $\left(<10^{16} \mathrm{~cm}^{-3} \mathrm{~s}^{-1}\right)$, $\Delta \sigma_{\mathrm{mc}} \gg \Delta \sigma_{\mathrm{exc}}$.

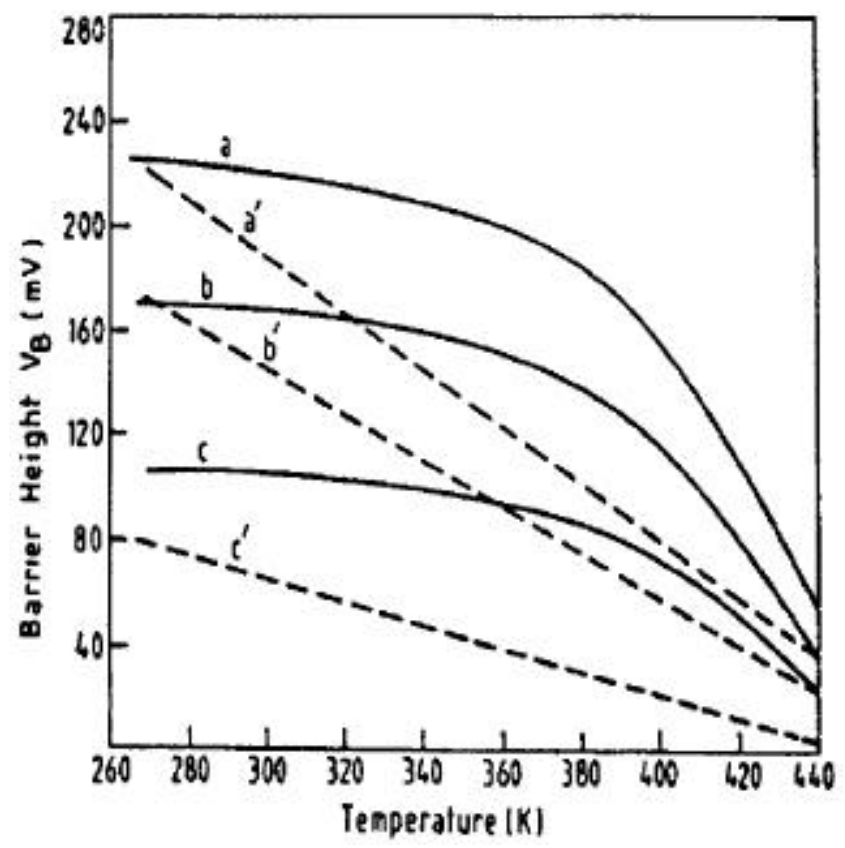

Figure 4. Estimated dependences of grain boundary barrier height on temperature for two different kinds of trap centres at grain boundaries (Singh et al 1985a).

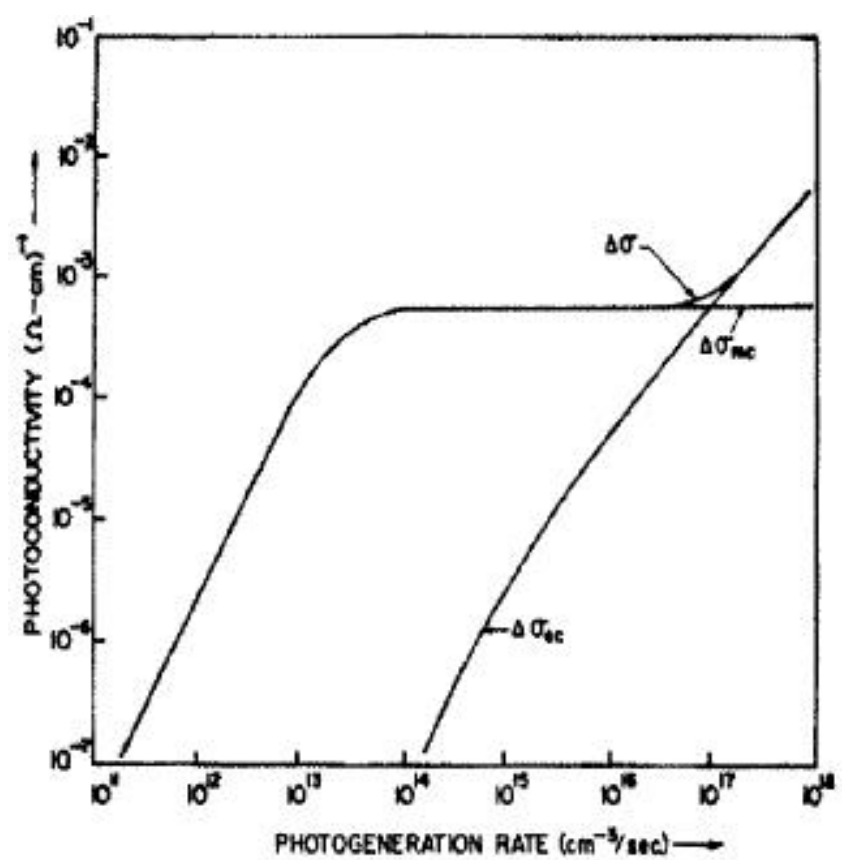

Figure 5. Photoconductivity of a polycrystalline silicon sample as a function of carrier generation rate (Singh et al 1986). 
Figure 6 shows the variation of resistivity, $\rho$, with reciprocal temperature under dark and under illumination with white light of different intensity values for a polycrystalline silicon specimen $\mathrm{S} 1$ of grain size, $1.7 \mathrm{~mm}$. The portions of the curves on the right hand side of the peak value shows an increasingly large decrease in $\rho$ under illumination with decrease in temperature, $T$. For $P_{\text {in }}=50 \mathrm{~mW} / \mathrm{cm}^{2}$ at $T=300 \mathrm{~K}$, the values of $\rho, \sigma$ and $\Delta \sigma_{0}$ are given in table 1 for sample, $\mathrm{S} 1$ and another sample, S2. It may be noted that $\Delta \sigma \ll \sigma_{0}$ is not valid.

\subsection{Photoconductivity decay behaviour}

Substituting (8-10) into (2) we get

$$
\Delta V(t)=-\frac{I d}{A \sigma_{0}}\left\{\frac{\Delta \sigma_{\mathrm{n}}}{\mu_{\mathrm{n}}}+\left(1+\frac{1}{b}\right) \frac{\Delta p}{n_{0}}\right\},
$$

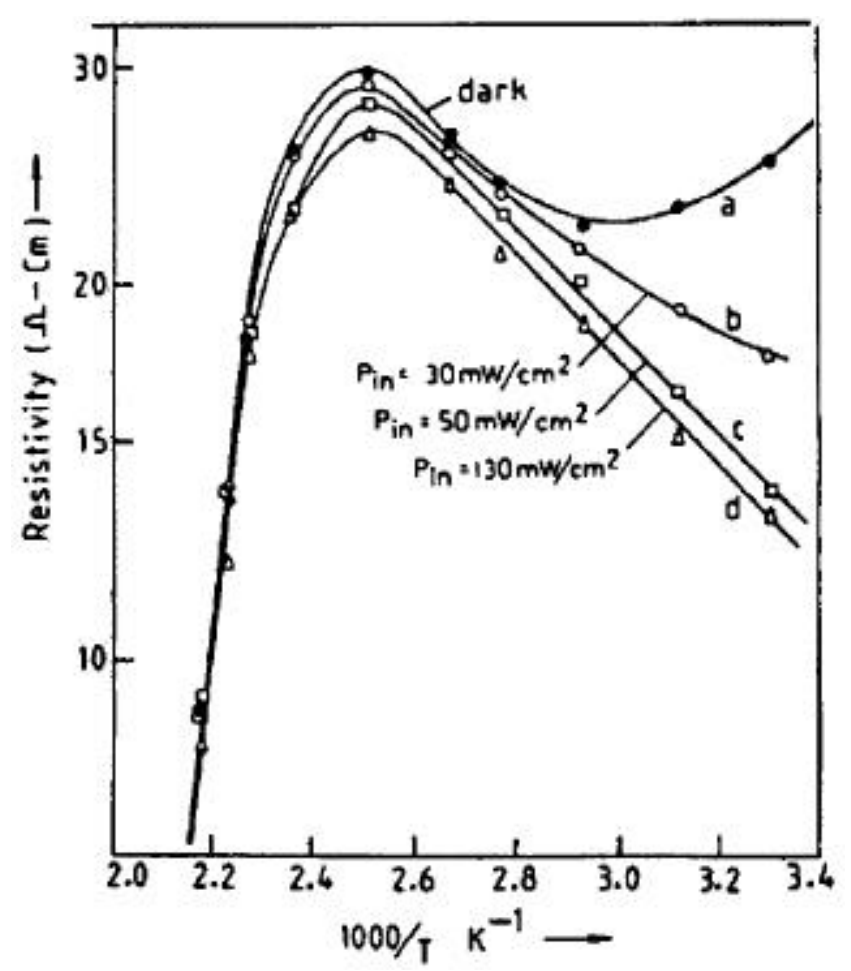

Figure 6. Variation of resistivity of an $n$-type Si sample S1 having grain size, $d_{\mathrm{g}}=1.7 \mathrm{~mm}$ (Singh et al 1985a). where $b$ is a constant given by $\mu_{\mathrm{n}} / \mu_{\mathrm{p}}$. In fact (13) represents a general case and is applicable for both multi and single crystalline silicon.

Even under low level conditions the change in majority carrier mobility in $n$-type multicrystalline silicon leads to a large change in $\Delta \sigma$ and therefore, $\Delta \sigma \ll \sigma_{0}$ is not satisfied. As a result the voltage signal is not represented by excess carrier alone and decays more slowly.

If $\left(\Delta \mu_{n} / \mu_{n}\right) \ll \Delta p / n_{0}$ or if $\Delta \mu_{n}=0$, as in case of single crystal silicon, then (13) will reduce to

$$
\Delta V(t)=-\frac{I d}{A \sigma_{0}}\left(1+\frac{\mu_{\mathrm{po}}}{\mu_{\text {no }}}\right) \frac{\Delta p}{n_{0}} .
$$

Considering the time dependence of $\Delta p$ during PCD, we have

$$
\Delta p_{(t)}=\Delta p_{(t=0)} \exp (-t / \tau)
$$

which leads to

$$
\Delta V_{(t)}=\Delta V_{(t=0)} \exp (-t / \tau)
$$

In the region where $\Delta \sigma_{\mathrm{mc}} \gg \Delta \sigma_{\text {exc }},\left(\Delta \mu_{\mathrm{n}} / \mu_{\mathrm{n}}\right) \gg \Delta p / n_{0}$ is valid, the PCD is controlled by decay of majority carrier mobility and under such conditions (14) reduces to

$$
\Delta V(t)=-\frac{I d}{A \sigma_{0}} \frac{\Delta \mu_{\mathrm{n}}}{\mu_{\mathrm{n}}} .
$$

In presence of grain boundary potential barrier, $\Delta \mu_{n} / \mu_{n}$ and $\Delta p$ are related through (6)

$$
\frac{\Delta \mu_{\mathrm{n}}}{\mu_{\mathrm{n}}}=\frac{\Delta p}{P_{0}+\Delta p}\left\{1+\frac{\mu_{0}}{\mu_{\mathrm{g}}} \exp \left(-\frac{q V_{\mathrm{Bo}}}{k T}\right)\right\}^{-1} .
$$

Substituting (18) in (17), the PCD is described as

$$
\Delta V_{(t)}=-\left(\frac{I d}{A \sigma_{0}}\right)\left\{\left(\frac{\Delta p}{p_{0}+\Delta p}\right)\left(1+\frac{\mu_{0}}{\mu_{\mathrm{g}}} \exp \frac{q V_{\mathrm{Bo}}}{k T}\right)^{-1}\right\}
$$

Therefore, considering the time dependence of terms on RHS, (19) can be expressed as

$$
\Delta V_{(t)}=-\Delta V_{(t=0)}\left\{\frac{\Delta P_{\mathrm{t}}}{P_{0}+\Delta P_{\mathrm{t}}}\right\}
$$

where $\Delta p_{(t)}$ is again given by (15).

Table 1. Values of various parameters for sample S1 obtained from figure 8. Similar values obtained for sample S2 are also listed.

\begin{tabular}{lccccc}
\hline Sample & $\begin{array}{c}\rho_{0} \\
(\Omega \mathrm{cm})\end{array}$ & $\begin{array}{c}\rho \\
(\Omega \mathrm{cm})\end{array}$ & $\begin{array}{c}\sigma_{0} \\
\left(\Omega^{-1} \mathrm{~cm}^{-1}\right)\end{array}$ & $\begin{array}{c}\sigma \\
\left(\Omega^{-1} \mathrm{~cm}^{-1}\right)\end{array}$ & $\begin{array}{c}\Delta \sigma \\
\left(\Omega^{-1} \mathrm{~cm}^{-1}\right)\end{array}$ \\
\hline S1 & 25 & 12 & 0.04 & 0.07 & $\begin{array}{c}0.03 \\
\left(\Delta \sigma<\sigma_{0}\right) \\
\text { S2 }\end{array}$ \\
\hline
\end{tabular}


Equation (20) shows that the voltage decay is not linearly related with $\Delta p_{\mathrm{t}}$ and therefore, decreases more slowly. It also shows that if $p_{0}$ is increased such that $p_{0} \gg \Delta p_{\mathrm{t}}$, then $\Delta V(t)$ will be proportional to $\Delta p_{\mathrm{t}}$ and decay signal will give true value of $\tau$. This can be done either by illuminating the sample with background light of sufficiently high intensity or by increasing $T$ so that $\Delta \mu_{\mathrm{n}} / \mu_{\mathrm{n}} \ll \Delta p / n_{0}$ and the mobility modulation becomes insignificant. Figures 7 and 8 show this behaviour.

\section{Photogeneration method}

Following Jain et al (1983) and Sharma et al (1986), we find that for most cases of interest, $w \gg d_{\mathrm{p}}$ where $w$ is the wafer thickness and $d_{\mathrm{p}}$ the accumulation layer $\left(p^{+}\right)$thickness, $L \gg L_{\lambda} \gg d_{\mathrm{p}}$, where $L$ is the diffusion length of electrons and $L_{\lambda}$ the reciprocal of the absorption coefficient, $\alpha_{\lambda}$, of the incident monochromatic light. For such cases the photo generated short circuit current density $\left(J_{\mathrm{SC}}\right)$ for illumination from the $p^{+}$side is related with the intensity $\left(P_{\text {in }}\right)$ of the incident light as

$$
J_{\mathrm{SC}}=\frac{q \lambda\left(1-R_{\lambda}\right) L L_{\lambda}}{h c\left(L^{2}-L_{\lambda}^{2}\right) \cos h(w / L)} P_{\mathrm{in}} .
$$

In the above equation, $R_{\lambda}$ is the reflectivity of the $p^{+}$ surface. For silicon, the above equation is satisfied well if $S$, the surface recombination velocity of electrons in the $p$-region near the accumulation layer is small, i.e.

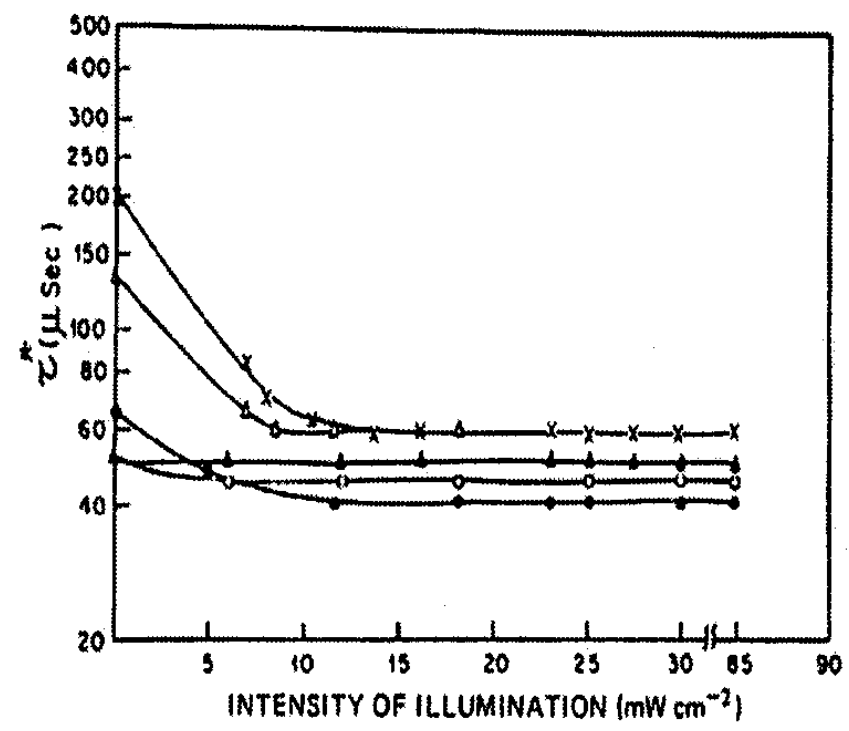

Figure 7. Variation of PCD time constant, $\tau^{*}$, with intensity of background illumination with white light for five specimen: $\times$, float zone recrystallized, twinned; $\bullet$, float zone recrystallized, small grain; 0 , float zone recrystallized, single grain; $\Delta$, Wacker Silso; and $\boldsymbol{\Delta}$, Czochralski single crystal (Singh et al 1985b).
$S<10^{3} \mathrm{~cm} / \mathrm{s}$ and the wavelength of the incident light is in the 750-900 $\mathrm{nm}$ range.

The $J_{\mathrm{SC}}$ vs $P_{\text {in }}$ curve is a straight line and its slope, $\Phi=d J_{\mathrm{SC}} / d P_{\text {in }}$ can be utilized to obtain $L$ using the equation

$$
L=w / \cos h^{-1}\left(\Phi_{\mathrm{m}} / \Phi\right),
$$

where $\Phi_{\mathrm{m}}=q(1-R) \lambda / h c$.

\subsection{Experimental}

A few polished $p$-Si wafers were cleaned in RCA solution and were passivated with a $\sim 100 \AA$ thermal $\mathrm{SiO}_{2}$ layer at $500^{\circ} \mathrm{C}$. Subsequently a semitransparent $\mathrm{Pd}$ layer of thickness $\sim 100 \AA$ was deposited on one side of wafer under vacuum. The other side was covered with a semitransparent Al layer of $\sim 1500 \AA$ thickness. We refer to this category of specimen preparation as $\mathrm{C} 1$. In some cases thermal $\mathrm{SiO}_{2}$ layer was not grown before metallization. We refer to this category as $\mathrm{C} 2$.

The PCG method was applied for measurement of photocurrent as a function of intensity of light for two wavelengths, viz. $800 \mathrm{~nm}$ and $900 \mathrm{~nm}$, using interference filters. The photocurrent was measured. The intensity of the incident light was determined using a reference silicon solar cell of known spectral response.

\section{Results and discussion}

Figure 9 shows the variation of short-circuit current density with intensity of illumination when light of wavelengths 800 and $900 \mathrm{~nm}$ was incident on the $p^{+}$side.

The reflectivity of the $p^{+}$surface was measured with Beckmann spectrophotometer and the slopes $\Phi$ and $\Phi_{\mathrm{m}}$ were determined. For $\Phi$, the linear portion of the $J_{\mathrm{SC}}$ vs

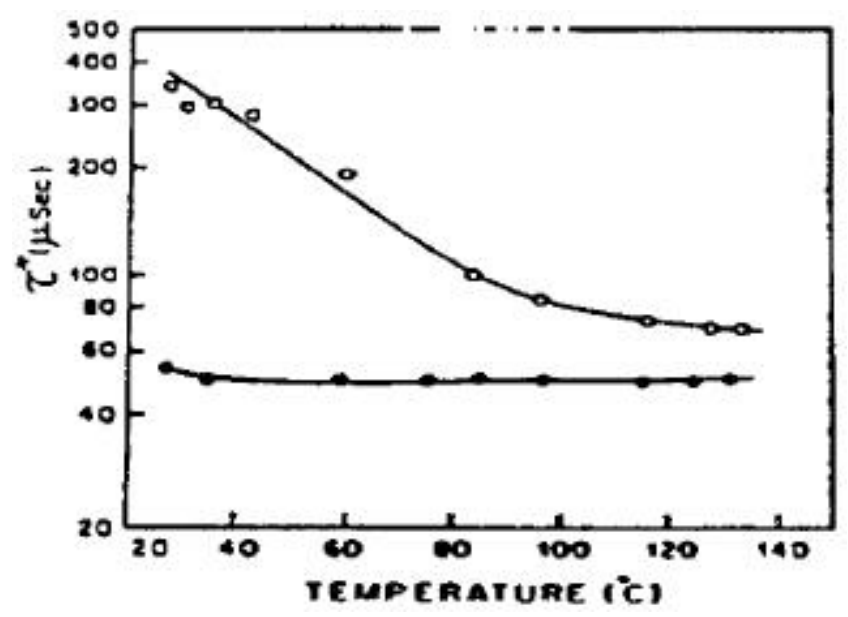

Figure 8. Variation of PCD time constant, $\tau^{*}$, with temperature under dark (0) and background illumination $(\bullet)$ (Singh et al 1985b). 
$P_{\text {in }}$ curve was used. The diffusion length, $L$, was obtained using (22).

The measurement of $L$ was also carried out using another method known as long wavelength spectral response (LWSR) method in the $800-1000 \mathrm{~nm}$ wavelength range.

Figure 10 shows $\lambda / J_{\text {SC }}$ vs $1 / \alpha_{\lambda}$ plot for $p^{+}-p-n^{+}$structure for illumination from the $n^{+}$side for the above wavelengths range. The value of $L$ was determined from the intercept of the straight line on the $1 / \alpha_{\lambda}$ axis.

Table 2 lists the values of various parameters required for determination of the diffusion length, $L$ by the PCG and LWSR methods. Both methods give nearly same value of $L$. With wafer having resistivity of $1 \Omega \mathrm{cm}$, $D_{\mathrm{n}}=25 \mathrm{~cm}^{2} \mathrm{~s}^{-1}$, lifetime was determined using $\tau=L^{2} / D_{\mathrm{n}}$ and was found to be 2.82 microsec.

Similar studies were done on specimen $\mathrm{C} 2$ and the values of $\tau$ were determined. The measurement on these

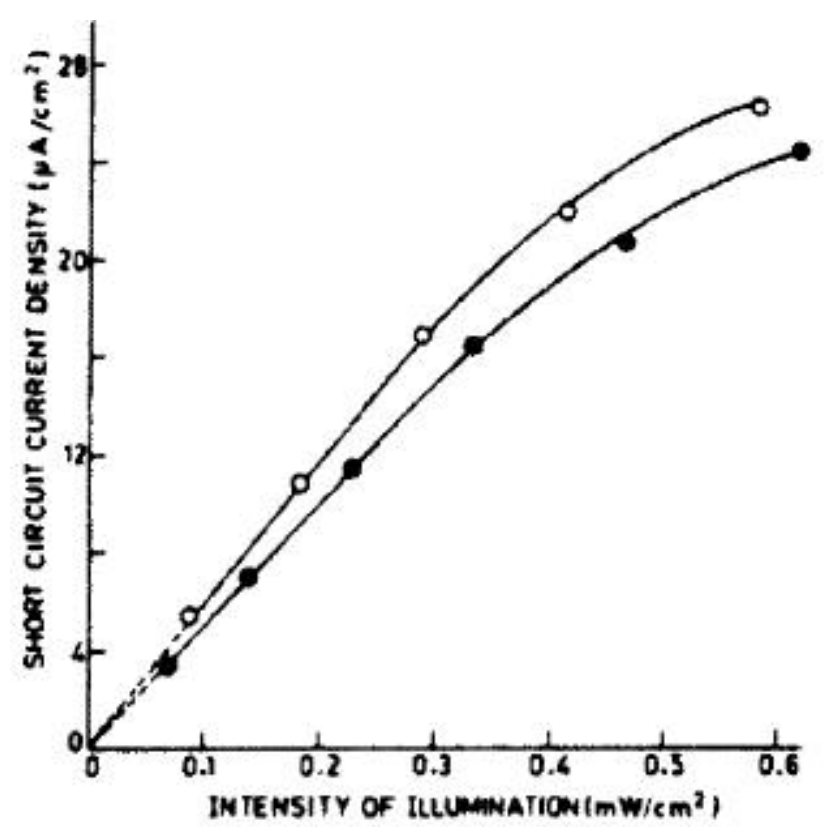

Figure 9. Short circuit current density vs intensity of illumination on the $p^{+}$side of $p^{+}-p-n^{+}$structure based silicon sample for $\lambda=800 \mathrm{~nm}$ and $\lambda=900 \mathrm{~nm}$ (specimen C1) (Sharma et al 1986). samples were carried out under two conditions: (i) by keeping the specimen unexposed to the atmosphere and (ii) exposing them to atmosphere. The exposure to atmosphere ambience deteriorates the quality of the $p^{+}-p-$ $n^{+}$structure and it gives lower value of $\tau$. This behaviour is shown in figure 11. It reveals that the measurement of $\tau$ should be carried out by keeping the samples protected as the measurements after exposure to atmosphere gives erroneous result. The cause of deterioration of the structure and the error associated in determining $\tau$ using PCG in such cases are being investigated.

\section{Conclusions}

In PCD method the light pulse should lead to a small change in $\Delta \sigma$ so that $\Delta \sigma \ll \sigma_{0}$ and the decay is governed essentially by the change in the excess carrier density, $\Delta p$, due to the light pulse. In case of polycrystalline samples, $\Delta \sigma \ll \sigma_{0}$ may not be valid due to the presence of the grain boundary potential barrier, $V_{\mathrm{B}}$, which controls

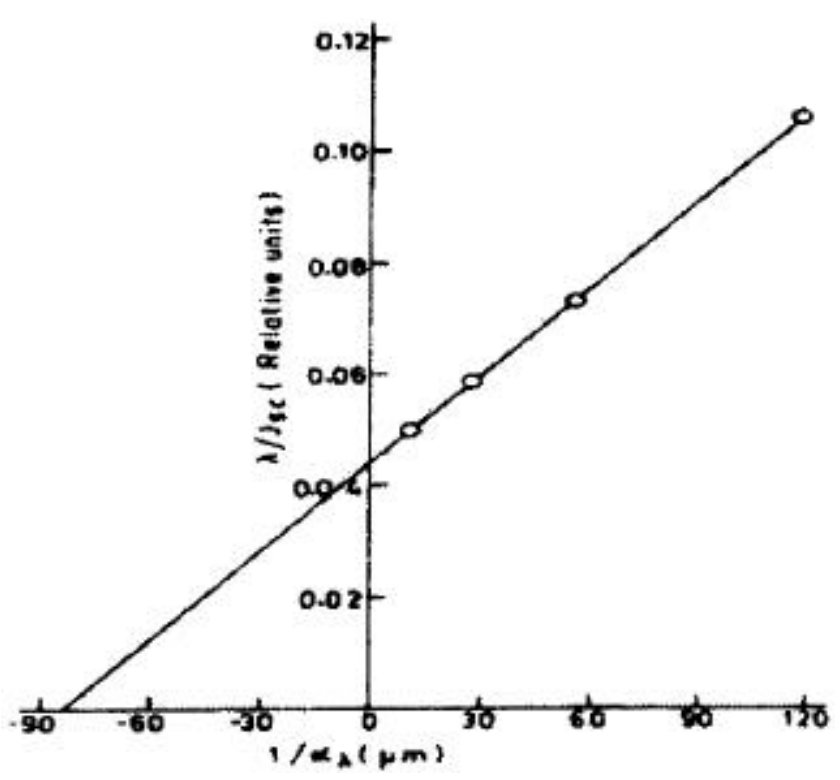

Figure 10. $\lambda / J_{\mathrm{SC}}$ vs $1 / \alpha_{\lambda}$ curves for $p^{+}-p-n^{+}$structure based silicon illuminated by monochromatic radiations in 800 $1000 \mathrm{~nm}$ range (specimen C1) (Sharma et al 1986).

Table 2. Values of various parameters required for determination of diffusion length, $L$, by the PCG and LWSR methods for specimen C1 $(w=220 \mu \mathrm{m})$.

\begin{tabular}{lcccccc}
\hline $\begin{array}{l}\lambda \\
(\mu \mathrm{m})\end{array}$ & $R_{\lambda}$ & $\begin{array}{c}\alpha_{\lambda}^{-1} \\
(\mu \mathrm{m})\end{array}$ & $\begin{array}{c}P_{\text {in }} \\
\left(\mathrm{mW} / \mathrm{cm}^{2}\right)\end{array}$ & $\begin{array}{c}\Phi \\
\left(\mathrm{AW}^{-1}\right)\end{array}$ & $\begin{array}{c}\text { PCG } \\
(\mu \mathrm{m})\end{array}$ & $\begin{array}{c}L \\
\text { LWSR } \\
(\mu \mathrm{m})\end{array}$ \\
\hline $0 \cdot 8$ & $0 \cdot 46$ & $10 \cdot 17$ & $0 \cdot 1-0 \cdot 6$ & 0.05 & 84 & $\ldots$ \\
$0 \cdot 9$ & $0 \cdot 46$ & $27 \cdot 55$ & $0 \cdot 1-0 \cdot 6$ & 0.06 & 86 & $\ldots$ \\
0.95 & $\cdots$ & $56 \cdot 18$ & $\cdots$ & $\cdots$ & $\cdots$ & 84 \\
$1 \cdot 0$ & $\cdots$ & $118 \cdot 12$ & $\cdots$ & $\cdots$ & $\cdots$ & $\cdots$ \\
\hline
\end{tabular}




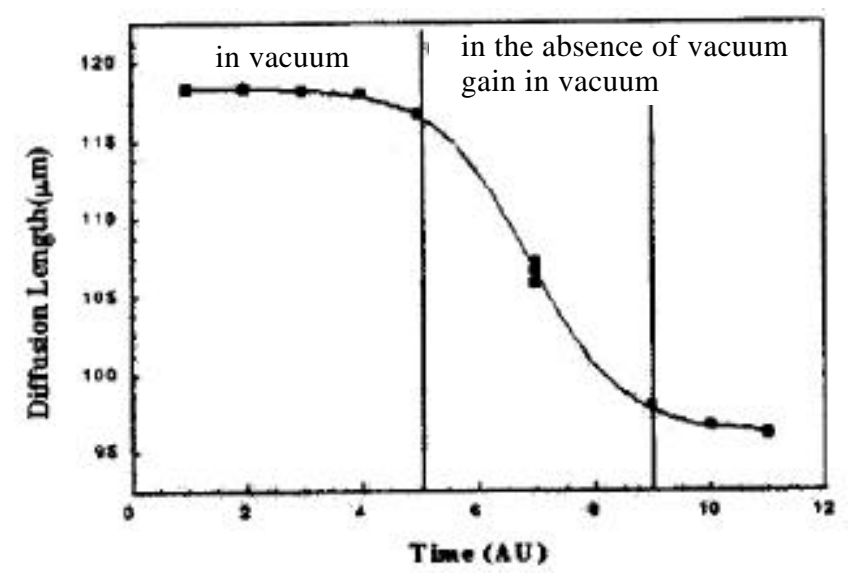

Figure 11. The effect of delayed measurement on the value of $\tau$ determined by PCG method (specimen $\mathrm{C} 2$ ). The middle part of the curve shows that exposure to atmosphere has a deleterious effect on the induced $p^{+}-p-n^{+}$structure leading to erroneous values of $\tau$. Time is in units of $12 \mathrm{~h}$.

majority carrier mobility. In presence of $V_{\mathrm{B}}$ the PCD constant $\tau^{*}$ is larger than the lifetime, $\tau$. PCD measurement with background illumination of specimen suppress $V_{\mathrm{B}}$ and then the decay constant of the voltage signal gives true lifetime.

The PCG method can be applied to a chemically and mechanically polished $p$-Si wafer for $\tau$ measurement without creating a $\mathrm{SiO}_{2}$ layer on them. However, the exposure of the induced $p^{+}-p-n^{+}$structure to atmosphere affects the results. To get reliable results, it is desired that the $\tau$ measurement on the induced $p^{+}-p-n^{+}$structure should be carried out in vacuum and soon after the creation of the induced structures.

\section{Acknowledgement}

The authors are thankful to Mr Ravi Kumar for his help in the specimen preparation.

\section{References}

Ciszek Ted F Silicon defect and impurity studies using controlled samples (USA: National Centre for Photovoltaics, NREL)

Citarella G, Von Aichberger S and Kunst M 2002 Mater. Sci. \& Eng. B91-B92 224

Jain G C, Singh S N and Kotnala R K 1983 Solar Cells 8239

Sharma S K, Singh S N, Chakravarty B C and Das B K 1986 J. Appl. Phys. 603550

Singh P K, Singh S N and Kishore R 1986 Appl. Phys. Lett. 48 127

Singh S N, Kishore R and Singh P K 1985a J. Appl. Phys. 57 2793

Singh S N, Kishore R and Arora N K 1985b Solar Cells 1413 